\title{
Digital Subtraction Angiography for CT Angiogram Negative Haemorrhages
}

\author{
Helen Wong, Luke Hodgson, Jillian Banfield, Jai J. S. Shankar
}

\begin{abstract}
Background: In patients with subarachnoid haemorrhage (SAH) and a negative finding on CT angiography (CTA), further imaging with digital subtraction angiography (DSA) is commonly performed to identify the source of bleeding. The purpose of this study was to investigate whether negative findings on CTA can reliably exclude aneurysms in patients with acute SAH. Methods: This retrospective study identified all DSAs performed between August 2010 and July 2014 within our institution. CT angiography was performed with a 64-section multidetector row CT scanner. Only DSAs from patients with confirmed SAH and a negative CTA result were included in the final analyses. A fellowship-trained neuroradiologist reviewed the imaging results. Results: Of the 857 DSAs, 50 (5.83\%) were performed in 35 patients with CTA-negative SAH. Of the 35 patients, three $(8.57 \%)$ had positive findings on the DSA. In one patient, suspicious dissection of the extra- and intra-cranial segment of the right vertebral artery could not be confirmed even in retrospect. In the second patient, the suspicious finding of tiny protuberance from the left paraclinoid internal carotid artery (ICA) on DSA did not change on follow-up and did not change patient's management. The third patient had a posterior inferior cerebellar artery aneurysm, which was not seen on the initial CTA owing to the incomplete coverage of the head on the CTA. Conclusion: In patients with SAH, negative findings on a technically sound CTA are reliable in ruling out aneurysms in any pattern of SAH or no blood on CT. Our observations need to be confirmed with larger prospective studies.
\end{abstract}

RÉSUMÉ: Effectuer un examen d'angiographie numérique avec soustraction chez des patients victimes d'une hémorragie méningée pour lesquels un examen de tomodensitométrie a donné des résultats négatifs. Contexte: Dans le cas de patients victimes d'hémorragie méningée pour lesquels un examen d'angiographie par tomodensitométrie donne des résultats négatifs, l'obtention d'images additionnelles au moyen de l'angiographie numérique avec soustraction (ANS) est couramment envisagée afin de déterminer l'origine du saignement. L'objectif de cette étude est donc d'examiner dans quelle mesure des résultats négatifs à un examen d'angiographie par tomodensitométrie peuvent exclure en toute certitude la présence d'anévrismes chez des patients victimes d'hémorragie méningée aigüe. Méthodes: Cette étude rétrospective a ainsi procédé à l'identification de tous les examens d'ANS réalisés au sein de notre établissement entre août 2010 et juillet 2014. Des examens d'angiographie par tomodensitométrie ont été ensuite effectués au moyen d'un tomodensitomètre à 64 barrettes de détecteurs. Ainsi, seuls les patients ayant subi un examen d'ANS et pour lesquels on a confirmé la présence d'une hémorragie méningée à la suite de résultats négatifs à un examen de tomodensitométrie ont été inclus dans le cadre de notre travail final d'analyse. Résultats: Sur les 857 examens d'ANS, $50(5,83 \%)$ ont été réalisés chez 35 patients victimes d'hémorragie méningée pour lesquels un examen d'angiographie par tomodensitométrie avait donné des résultats négatifs. De ces 35 patients, trois $(8,57 \%)$ ont donné à voir des résultats positifs à un examen d'ANS. Dans le cas d'un patient, une dissection suspecte des segments extra-crânien et intracrânien de l'artère vertébrale droite n'a pas pu être confirmée, et ce, même ultérieurement. Dans le cas d'un second patient ayant subi un examen d'ANS, une minuscule protubérance suspecte sur l'artère carotide interne paraclinoïde gauche n'avait pas évolué au moment d'un suivi. Fait à souligner, une telle découverte n'a pas modifié la prise en charge du patient. Enfin, on a pu détecter chez le troisième patient un anévrisme ayant affecté l'artère cérébelleuse postérieure-inférieure, ce qui n'avait pas été observé lors du premier examen de tomodensitométrie, la tête du patient n'ayant pas été couverte complètement par l'appareil. Conclusion: Dans le cas de patients victimes d'une hémorragie méningée, des résultats négatifs obtenus au moyen d'un tomodensitomètre techniquement bien ajusté permettent d'exclure de façon fiable la présence d'anévrismes pour tous les cas d'hémorragie méningée ou lorsqu'un tomodensitomètre ne révèle pas de saignement. Cela dit, nos observations doivent encore être confirmées par des études prospectives de plus grande envergure.

Keywords: Digital subtraction angiograph, Subarachnoid haemorrhages

doi:10.1017/cjn.2018.75

Can J Neurol Sci. 2018; 45: 522-526

\section{INTRODUCTION}

The most common causes of non-traumatic subarachnoid haemorrhage $(\mathrm{SAH})$ are ruptured aneurysms and have very poor natural history. ${ }^{1}$ Digital subtraction angiography (DSA) is commonly performed after CT angiography (CTA) when a patient is suspected to have an SAH to rule out intracranial aneurysms. ${ }^{2}$ The most recent guideline from the American Heart and American
From the Department of Diagnostic Radiology, QEII Health Sciences Centre, Halifax, Nova Scotia, Canada (JJSS, HW, LH, JB).

Received January 16, 2018. Revisions Submitted January 16, 2018. Date of ACCEPTANCE APRIL 12, 2018.

Correspondence to: Jai J. S. Shankar, Department of Diagnostic Radiology, QEII Health Sciences Centre, 1796 Summer Street Halifax, Nova Scotia, Canada B3H 3A7.

Email: shivajai1@gmail.com 
Table 1: Demographic details of the patients with CT angiography (CTA)-negative subarachnoid haemorrhage (SAH)

\begin{tabular}{l|c}
\hline Characteristics & $\boldsymbol{n}$ \\
\hline Number of patients with DSA and CTA-negative SAH & 35 \\
\hline Male:female & $17: 18$ \\
\hline Median age (range) & 57 (24-79) years \\
\hline Median time interval between CTA and DSA (range) & \\
\hline Pattern of SAH on plain CT head & 23 \\
\hline Perimesencephalic haemorrhage & 6 \\
\hline Aneurysmal pattern of SAH & 3 \\
\hline Peripheral SAH & 3 \\
\hline CT - ve and LP + ve SAH & \\
\hline
\end{tabular}

$-\mathrm{ve}=$ negative $;+\mathrm{ve}=$ positive DSA = digital subtraction angiography; $\mathrm{LP}=$ lumbar puncture.

Stroke Associations recommends using DSA for detecting aneurysms in patients with $\mathrm{SAH}^{3}$ The guidelines indicate that CTA can be used to guide decisions regarding aneurysm repair, but that DSA should be used when CTA is inconclusive. Despite these guidelines, clinicians and researchers are divided about the necessity of using DSA to detect aneurysms after SAH. ${ }^{4-9}$ Up to $20 \%$ of SAH patients have no aneurysm detected on initial angiography imaging. ${ }^{10,11}$ Given that DSA is resource-intensive and invasive, CTA is increasingly being used to detect ruptured aneurysms following SAH. ${ }^{12}$

Imaging of head and neck vessels with CTA has improved since helical CT was introduced, and it provides high-resolution three-dimensional reconstructions and shorter acquisition time. ${ }^{4}$ In addition to these improvements in technology, radiologists' increasing familiarity with interpreting source images has increased the utility of CTA. ${ }^{2}$ Thus, interventional radiologists are able to diagnose patients more efficiently with suspected SAH because of enhancements in CTA imaging and training. Increased use of CTA, however, has not been accompanied by a matching decrease in DSA, resulting in multiple tests without gain in diagnostic yield. ${ }^{12}$ Given the improvements in CTA and the disadvantages of DSA, it is imperative to investigate whether a DSA is truly necessary for a negative SAH finding on a CTA. ${ }^{4}$ The purpose of this study was to review cases of suspected aneurysmal SAH at our institution to determine whether a CTA was sufficient to exclude a diagnosis of intracranial aneurysms in patients with suspected SAH.

\section{Materials ANd Methods}

\section{Data Collection}

The study was approved by our institutional research ethics board. In a retrospective study, all cerebral DSA performed from August 2010 to July 2014 for any indications were analysed. Subarachnoid haemorrhage was identified through positive finding on non-contrast computed tomography scans and/or lumbar punctures. Among those with confirmed SAH, only patients who had a negative CTA were selected for this study. Results from CTA with source images were compared with DSA.

\section{Image Acquisition and Analysis}

Following standard protocols, CTA was performed on 64-section multidetector CT scanners and DSA in a dedicated biplane neuroangiography unit (Axiom Artis, Siemens). All images were analysed on a Picture Archiving and Communication System (PACS) workstation. Retrieval of patient information and radiology reports were reviewed for patient selection. The CTA and DSA images of the patients with CTA-negative SAH were reviewed on PACS by a fellowship-trained neuroradiologist (JJSS) to confirm the diagnosis and to rule out any possibilities of dictation errors.

\section{RESULTS}

In total, 857 DSA were performed during the study period; 50 (5.83\%) DSA were performed in 35 patients who presented with SAH and had negative CTA for any possible aetiology of SAH. The patients' demographic information is summarised in Table 1. Of the 35 patients, only $3(8.57 \%)$ patients had positive findings on the DSA.

One patient presented with $\mathrm{SAH}$ in the pre-pontine and cerebellopontine cistern with CTA-negative for evidence of aneurysm (Figure 1). DSA showed irregularity in the V4 segment of the right vertebral artery. This was not obvious on CTA.

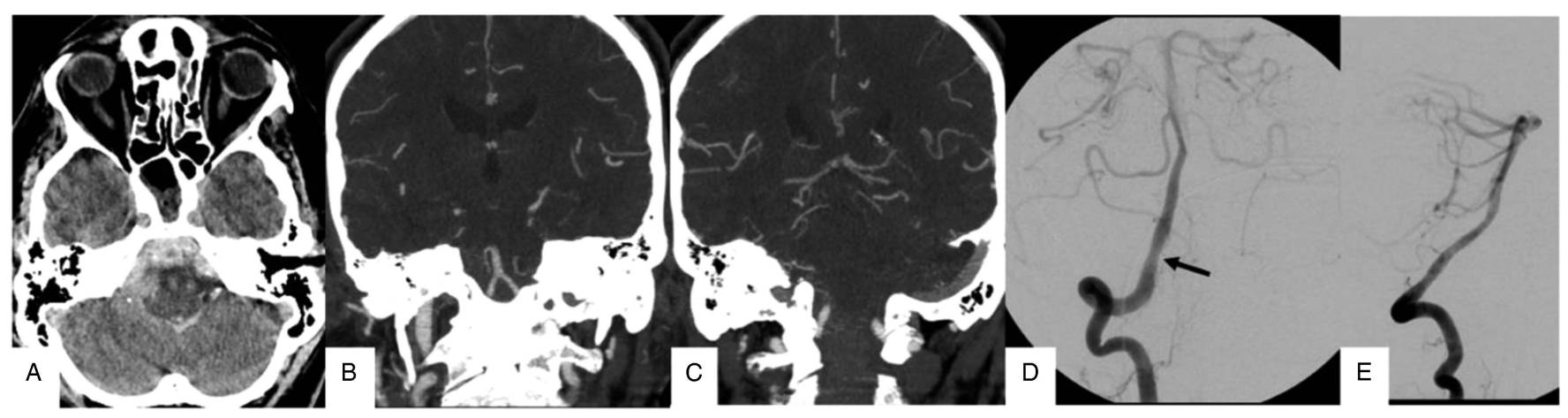

Figure 1: Patient presented with subarachnoid haemorrhage predominantly in the posterior fossa (A). CT angiogram did not show any source for this subarachnoid haemorrhage $(B, C)$. Digital subtraction angiography showed irregularity in the wall of the V4 segment of the right vertebral artery $(D)$, which was not very well seen on the lateral view $(E)$. In the absence of any other source and owing to the proximity of this irregularity to the subarachnoid haemorrhage, this was diagnosed as dissection of the vertebral artery. No other imaging evidence of dissection was seen. 


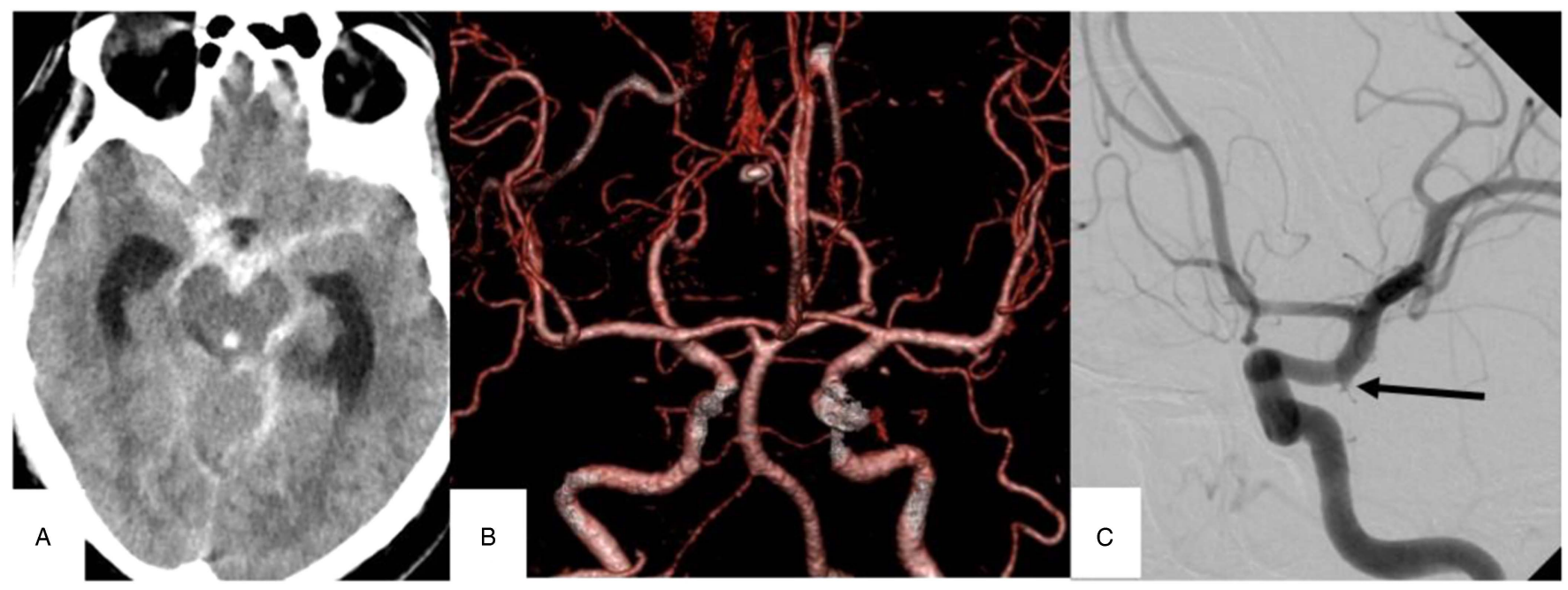

Figure 2: Patient had subarachnoid haemorrhage predominantly in the suprasellar and interpeduncular cistern more so on the right side (A). CT angiogram did not show any source of the subarachnoid haemorrhage (B). Digital subtraction angiogram showed a suspicious finding of a tiny infundibulum (arrow) from the contralateral (left) paraclinoid ICA (C). This remained stable on the 3rd follow-up for subarachnoid haemorrhage and was thought not to be the source of subarachnoid haemorrhage.

In the absence of any other obvious aetiology and owing to the presence of maximum SAH around this area, this was thought to be a dissection. This was not obvious on CTA. This finding was equivocal and could have been a focal vasospasm from the presence of the SAH. Vasospasm on day 1 after SAH is extremely rare.

The second patient had SAH in the pre-pontine cistern with intra-ventricular extension with negative CTA for any evidence of aneurysm. On DSA, the only suspicious finding was a tiny ( $1.5 \mathrm{~mm}$ in maximum diameter) outpouching from the left paraclinoid ICA (Figure 2). The appearance favoured an infundibular origin of a hypoplastic posterior communicating artery. However, a tiny, sessile aneurysm could not be excluded. This finding did not show any change even on the third follow-up DSA. The patient did not receive any treatment for this finding, did not have a re-bleed and made an excellent recovery.

The third patient was referred to us from another institution with the initial diagnostic CT and CTA showing SAH but no aneurysms. The patient underwent DSA in our institution that demonstrated a small posterior inferior cerebellar artery aneurysm. This patient underwent another CTA in our institution before undergoing coiling, which demonstrated the aneurysm of exactly the same shape and size. In retrospect, the initial CTA did not cover the region of the aneurysm (Figure 3 ).

\section{Discussion}

In this retrospective study comparing CTA with the criterion standard of DSA, we demonstrated the relative futility of DSA in patients with CTA-negative SAH. Of the 35 patients with CTAnegative $\mathrm{SAH}$, three were reported to have positive findings on follow-up DSA. Dissection was suspected but could not be confirmed in the first patient. A tiny, sessile aneurysm could not be ruled out in the second case, but the patient received no treatment for an aneurysm and made a full recovery. In the third patient, a posterior inferior cerebellar artery aneurysm was not seen initially because the initial CTA did not cover the area of the brain where the aneurysm was located. When another CTA was performed immediately after the DSA, it showed the aneurysm very well. The final case underscores the importance of having properly trained professionals who meticulously follow standard protocols in optimising the use of CTA.

Our findings are consistent with earlier studies that report high sensitivity (97\%-100\%) of CTA for detecting intracranial aneur-
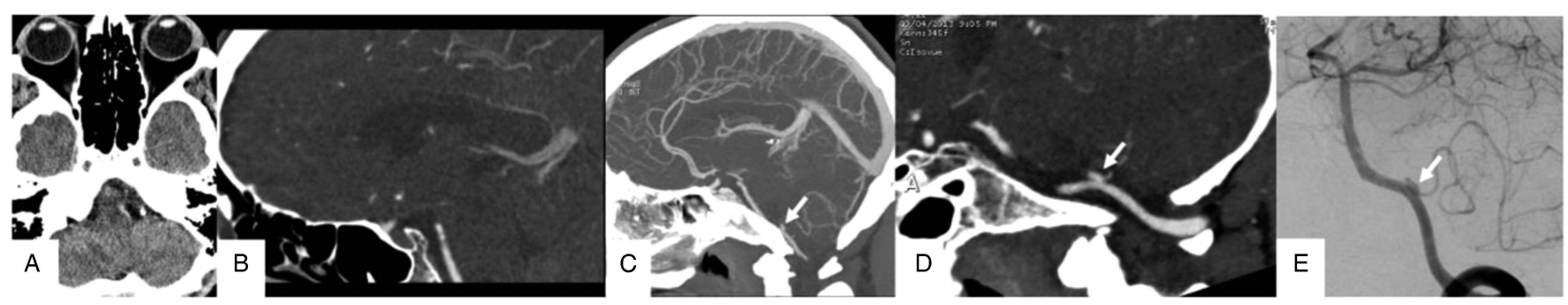

Figure 3: Patient had a small subarachnoid haemorrhage in the left cerebellopontine angle cistern (A). CT angiogram performed in an outside institution did not show any source of this subarachnoid haemorrhage (B), but this CT angiogram did not even cover the area of focal subarachnoid haemorrhage. CT angiogram repeated in our institution covering the whole brain $(C)$ showed a left posterior inferior cerebellar artery origin aneurysm $(C$, arrow). This aneurysm (arrow) seen on the reconstructed image $(D)$ looked exactly similar in size and shape to what was seen on the digital subtraction angiography (E, arrow). 
ysms. ${ }^{13-15}$ Similarly, results from a large systematic review and meta-analysis comprising 50 studies calculated the pooled sensitivity and specificity of CTA in the diagnosis of cerebral aneurysms in SAH to be $98 \%$ and $100 \%$, respectively. ${ }^{16}$ In lieu of DSA, the aforementioned evidence provides strong support for CTA to serve as the sole diagnostic imaging modality for intracranial aneurysm detection within the SAH population.

However, a recent retrospective analysis of 643 patients suggests that CTA accuracy may be lower than presented in earlier investigations. Philipp et $\mathrm{al}^{17}$ reported sensitivity of CTA to be $70.7 \%$ for overall $\mathrm{SAH}, 57.6 \%$ for very small aneurysms $(<5 \mathrm{~mm})$ and $45 \%$ for internal carotid artery aneurysms. As acknowledged by the authors, this was possibly a result of variability in imaging data relating to methods, locations, technology, time frame and expert staff in the study. ${ }^{17}$ The legitimacy of CTA in the diagnosis of cerebral aneurysms in patients with SAH has been challenged in the past. In addition to small aneurysms, previous studies have highlighted that CTA struggles with detecting aneurysms located adjacent to the bone/skull base or at a tortuous vessel loop. ${ }^{6,18-20}$

We argue that the sensitivity of CTA may have been underestimated in some studies owing to misinterpretation of imaging scans potentially because of the lack of observer experience. Earlier investigations have shown that the accuracy of CTA detection of intracranial aneurysms can improve as the readers gain more experience or exposure. ${ }^{21,22}$ One study reported sensitivities for CTA increasing from $69 \%$ on initial aneurysms $(n=13)$ to $84 \%$ on subsequent aneurysms $(n=13) .{ }^{22}$ Similarly, neuroradiologists have been found to consistently perform better than non-neuroradiologists in detecting intracranial aneurysms via CTA. ${ }^{23}$ This underscores the importance of both, the use of correct protocol with modern CTA technology and increasing expertise with the interpretation of these images, in increasing the capability in depicting intracranial aneurysms on noninvasive CTA compared with DSA. For example, in a prospective study of 133 patients and five independent reviewers of images, the sensitivity for aneurysms $<3 \mathrm{~mm}$ was reported to be greater for the 64-slice CTA $(96.3 \%)$ compared with the DSA $(85.2 \%) .^{24}$

Although DSA has long been regarded as the golden standard, an increasing number of studies show that DSA is unnecessary, invasive and costly. $^{8}$ Specifically, we propose that a DSA is unnecessary for the diagnosis of patients with SAH when a thorough CTA with appropriate protocol and review returns negative findings. On the basis of the present study, if CTA identifies no aneurysm in patients with $\mathrm{SAH}$, then it can be assumed that the origin of the SAH is non-aneurysmal in nature. Nevertheless, diagnostic accuracy is dependent on the physicians who interpret the images. As radiologists gain more experience with the technology, the advantages of CTA over DSA increase. ${ }^{2}$ Trusting such negative findings on CTA can expedite further management.

\section{Limitations}

The limitations of this study must be considered when interpreting its findings. Specifically, the retrospective nature of the study design and its potential for selection bias may limit the generalisability of its results. Given our small sample size, additional studies may be required to fully elucidate CTA diagnostic capabilities related to SAH.

\section{CONCLUSION}

In patients with $\mathrm{SAH}$, negative findings on a technically sound CTA are reliable in ruling out aneurysms in any pattern of SAH or no blood on CT. Our observations need to be confirmed with larger prospective studies.

\section{ACKNOWLEDGEMENT}

The authors acknowledge the Radiology Research Foundation of Dalhousie University for their funding of the summer research student grant.

\section{Financial Support}

This study was supported by funding provided by our institution's Radiology Research Foundation.

\section{CONFLICTS OF INTEREST}

The authors report no conflict of interest concerning the materials or methods used in this study or the findings specified in this paper.

\section{Statement of Authorship}

JJSS participated in the conception and design of the study. LH collected the raw data. LH and JJSS analysed and interpreted the data. JJSS was responsible for statistical expertise. JJSS, HW and JB were responsible for initial drafting of the manuscript. JJSS, $\mathrm{HW}, \mathrm{LH}$ and JB were involved in critically reviewing the draft manuscript and will be participating in revisions of the final manuscript.

\section{Disclosures}

All authors declare that they have nothing to disclose.

\section{REFERENCES}

1. van Gijn J, Rinkel GJ. Subarachnoid haemorrhage: diagnosis, causes and management. Brain J Neurol. 2001;124:249-78.

2. Thaker NG, Turner JD, Cobb WS, et al. Computed tomographic angiography versus digital subtraction angiography for the postoperative detection of residual aneurysms: a single-institution series and meta-analysis. J Neurointerventional Surg. 2012;4:21925.

3. Connolly ES, Rabinstein AA, Carhuapoma JR, et al. Guidelines for the management of aneurysmal subarachnoid hemorrhage: a guideline for healthcare professionals from the American Heart Association/American Stroke Association. Stroke. 2012;43:171137.

4. Agid R, Andersson T, Almqvist $\mathrm{H}$, et al. Negative CT angiography findings in patients with spontaneous subarachnoid hemorrhage: when is digital subtraction angiography still needed? AJNR. 2010;31:696-705.

5. Heit JJ, Pastena GT, Nogueira RG, et al. Cerebral angiography for evaluation of patients with CT angiogram-negative subarachnoid hemorrhage: an 11-year experience. AJNR. 2016;37: 297-304.

6. Kallmes DF, Layton K, Marx WF, et al. Death by nondiagnosis: why emergent CT angiography should not be done for patients with subarachnoid hemorrhage. AJNR. 2007;28:1837-8.

7. Lim LK, Dowling RJ, Yan B, et al. Can CT angiography rule out aneurysmal subarachnoid haemorrhage in CT scan-negative subarachnoid haemorrhage patients? J Clin Neurosci. 2014;21: 191-3.

8. Jabbarli R, Shah M, Taschner C, et al. Clinical utility and costeffectiveness of CT-angiography in the diagnosis of nontraumatic subarachnoid hemorrhage. Neuroradiology. 2014;56:817-24. 
9. MacKinnon AD, Clifton AG, Rich PM. Acute subarachnoid haemorrhage: is a negative CT angiogram enough? Clin Radiol. 2013;68:232-8.

10. Jadhav AP, Jovin TG. Vascular imaging of the head and neck. Semin Neurol. 2012;32:401-10.

11. Rinkel GJ, van Gijn J, Wijdicks EF. Subarachnoid hemorrhage without detectable aneurysm. A review of the causes. Stroke. 1993;24:1403-9.

12. McDonald JS, Kallmes DF, Lanzino G, et al. Use of CT angiography and digital subtraction angiography in patients with ruptured cerebral aneurysm: evaluation of a large multihospital data base. AJNR. 2013;34:1774-7.

13. Kershenovich A, Rappaport ZH, Maimon S. Brain computed tomography angiographic scans as the sole diagnostic examination for excluding aneurysms in patients with perimesencephalic subarachnoid hemorrhage. Neurosurgery. 2006;59:798-801; discussion 801-802.

14. Kelliny M, Maeder P, Binaghi S, et al. Cerebral aneurysm exclusion by $\mathrm{CT}$ angiography based on subarachnoid hemorrhage pattern: a retrospective study. BMC Neurol. 2011;11:8.

15. Prestigiacomo CJ, Sabit A, He W, et al. Three dimensional CT angiography versus digital subtraction angiography in the detection of intracranial aneurysms in subarachnoid hemorrhage. J Neurointerventional Surg. 2010;2:385-9.

16. Westerlaan HE, van Dijk JMC, van Dijk MJ, et al. Intracranial aneurysms in patients with subarachnoid hemorrhage: CT angiography as a primary examination tool for diagnosis- systematic review and meta-analysis. Radiology. 2011;258: 134-45.

17. Philipp LR, McCracken DJ, McCracken CE, et al. Comparison between CTA and digital subtraction angiography in the diagnosis of ruptured aneurysms. Neurosurgery. 2017;80:769-77.

18. Haider AS, Gottlich C, Khahera A, et al. The importance of catheter angiography in computed tomography angiography-negative subarachnoid hemorrhage. Cureus. 2017:9.

19. Jayaraman MV, Haas RA, Do HM, et al. Should CT angiography be routinely used in patients suspected of having aneurysmal subarachnoid hemorrhage? No! Radiology. 2009;254:314-5.

20. Moran CJ. Aneurysmal subarachnoid hemorrhage: DSA versus CT angiography - is the answer available? Radiology. 2011;258:15-7.

21. Pedersen HK, Bakke SJ, Hald JK, et al. CTA in patients with acute subarachnoid haemorrhage. A comparative study with selective, digital angiography and blinded, independent review. Acta Radiol Stockh Swed 1987. 2001;42:43-9.

22. Jayaraman MV, Mayo-Smith WW, Tung GA, et al. Detection of intracranial aneurysms: multi-detector row CT angiography compared with DSA. Radiology. 2004;230:510-8.

23. White PM, Wardlaw JM, Lindsay KW, et al. The non-invasive detection of intracranial aneurysms: are neuroradiologists any better than other observers? Eur Radiol. 2003;13:389-96.

24. Xing W, Chen W, Sheng J, et al. Sixty-four-row multislice computed tomographic angiography in the diagnosis and characterization of intracranial aneurysms: comparison with 3D rotational angiography. World Neurosurg. 2011;76:105-13. 